\title{
BP-1-102 exerts an antitumor effect on the AGS human gastric cancer cell line through modulating the STAT3 and MAPK signaling pathways
}

\author{
XIAOXIA JIANG ${ }^{1,2}$, JIAN TANG $^{1}$, MENGJIE WU $^{1,2}$, SHITU CHEN $^{1,2}$, ZHENZHEN XU $^{1,2}$, \\ HAIYONG WANG ${ }^{1,2}$, HAOHAO WANG ${ }^{1,2}$, XIONGFEI YU ${ }^{1}$, ZHONGQI LI ${ }^{1}$ and LISONG TENG ${ }^{1,2}$ \\ ${ }^{1}$ Cancer Center, The First Affiliated Hospital, College of Medicine, Zhejiang University; \\ ${ }^{2}$ Key Laboratory of Precision Diagnosis and Treatment for Hepatobiliary and Pancreatic Tumor of Zhejiang Province, \\ Hangzhou, Zhejiang 310003, P.R. China
}

Received May 19, 2018; Accepted January 4, 2019

DOI: $10.3892 / \mathrm{mmr} .2019 .9892$

\begin{abstract}
BP-1-102, a novel inhibitor of signal transducer and activator of transcription 3 (STAT3), exhibits significant antitumor effects in several malignancies in vitro and in vivo. However, its role in gastric cancer (GC) remains to be elucidated. In the present study, the effect and potential molecular mechanisms of BP-102 in human GC cell lines were investigated. The results showed that BP-1-02 dose-dependently inhibited the proliferation of AGS cells, whereas it had little effect on HGC-27 cells. Flow cytometric analysis indicated that BP-1-102 induced apoptosis, but had minimal effect on cell cycle distribution. In addition, cells treated with BP-1-102 demonstrated markedly suppressed migration and invasion capacities. Western blot analysis revealed that BP-1-102 inhibited the phosphorylation of STAT3 and its target genes, including c-Myc, cyclin D1 and survivin, in a time- and dose-dependent manner. Furthermore, it was found that BP-1-102 induced the phosphorylation of c-Jun N-terminal kinase and p38 mitogen-activated protein kinase (MAPK) and inhibited the activation of extracellular signal-related kinases. Taken together, these results demonstrated that BP-1-102 may be a potent antitumor agent that acts through modulating the STAT3 and MAPK signaling pathways in GC cells.
\end{abstract}

\section{Introduction}

Gastric cancer (GC) is one of the most common causes of cancer-associated mortality worldwide and is a major health

Correspondence to: Dr Lisong Teng, Cancer Center, The First Affiliated Hospital, College of Medicine, Zhejiang University, 79 Qingchun Road, Hangzhou, Zhejiang 310003, P.R. China E-mail: 1steng@zju.edu.cn

Key words: BP-1-102, gastric cancer, target therapy, signal transducer and activator of transcription 3 pathway, mitogen-activated protein kinase pathway problem in China with an increasing incidence and mortality rate according to a survey in $2015(1,2)$. Although advances have been made in the detection and clinical treatment of GC in previous decades, marked variation in survival rates are found between patients diagnosed at different tumor stages. Patients diagnosed during the later stage often have metastatic disease and are no longer able to receive surgical treatment, leaving chemotherapy as the only available option (3). However, chemotherapy is often associated with a low response rate, high toxicity and drug resistance in a large number of patients (4-6). Therefore, there is an urgency for identifying and developing novel compounds to optimize therapeutic options and improve the prognosis of patients with GC.

Signal transducer and activator of transcription 3 (STAT3) is the most studied member of the STAT family and is constitutively activated in various malignancies, including GC (7). Under normal physiological conditions, cells exhibit transient STAT3 phosphorylation, which lasts for only a relatively short period of time; however, once the tumor-related signaling pathways are dysregulated, this process becomes constitutive (8). Following activation, STAT3 undergoes phosphorylation-induced dimerization, nuclear translocation and binding to its target genes, leading to the transcriptional activation of downstream target genes that regulate tumor cell proliferation and progression $(3,9)$. There is substantial evidence demonstrating that the phosphorylation of STAT3 is abnormally activated in GC and that the constitutive activation of STAT3 is positively correlated with a poor prognosis and metastasis, indicating that it may serve as a negative prognostic factor (7,10-12). As a consequence, inhibitors targeting the activation of STAT3 offer promise in suppressing cancer proliferation and mobility and are being widely investigated in GC and several other types of cancer that contain activated STAT3 $(13,14)$. However, the majority of STAT3 inhibitors fail to demonstrate satisfactory ability to suppress tumor growth and/or have high toxicity (15). Therefore, it remains important to identify novel inhibitors of STAT3 activation that are effective in treating GC, while producing minimal side effects.

In addition, the dysregulation of mitogen-activated protein kinase (MAPK) signaling is closely associated with cell 
growth, progression and apoptosis. The three most widely studied MAPKs are extracellular signal-related kinases (ERKs), p38 MAPKs and c-Jun NH2-terminal kinases (JNKs) (16). Increasing evidence suggests that the activation of ERK, p38 and JNK MAPK signaling is involved in cancer initiation and progression, indicating that they may be promising therapeutic targets $(17,18)$.

BP-1-102 was designed as a STAT3 inhibitor and is an analog of S3I-201, which functions by disrupting STAT3 homodimerization (19). BP-1-102 inhibits the STAT3 Src homology 2 (SH2) domain, binds STAT3 with an affinity of $504 \mathrm{nM}$, and disrupts STAT3:STAT3 complex formation with a half maximal inhibitory concentration $\left(\mathrm{IC}_{50}\right)$ of $6.8 \mu \mathrm{M}$, which is a notable improvement from that of S3I-201 $\left(\mathrm{IC}_{50}=86 \mu \mathrm{M}\right)$; however, BP-1-102 has minimal or no effect on other STATs, including STAT1 and STAT5 (20,21). The antitumor effect of BP-1-102 has been evaluated in human pancreatic, breast, prostate, liver and lung cancer in vitro, and in human breast and non-small cell lung tumor xenografts in vivo through either tail vein or oral administration $(20,21)$; however, its role in GC has not been reported. Therefore, in the present study, in vitro experiments were conducted to investigate whether BP-1-102 exerts an antitumor effect on GC cells with constitutively activated STAT3 and to examine the molecular mechanisms involved.

\section{Materials and methods}

Cell lines and culture conditions. Five human gastric cancer cell lines (AGS, HGC-27, MKN28, MGC803 and SGC7901) were obtained from the Institute of Cellular Biology (Chinese Academy of Science, Shanghai, China) and cultured in RPMI-1640 medium (Gibco; Thermo Fisher Scientific, Inc., Waltham, MA, USA) supplemented with $10 \%$ fetal bovine serum (FBS; Gibco; Thermo Fisher Scientific, Inc.) and $1 \%$ penicillin/streptomycin (Thermo Fisher Scientific, Inc.) in a humidified $37^{\circ} \mathrm{C}$ incubator with $5 \% \mathrm{CO}_{2}$.

Reagents and antibodies. The novel STAT3 inhibitor BP-1-102 was obtained from Selleck Chemicals, LLC (Houston, TX, USA) and dissolved in sterile dimethyl sulfoxide (DMSO; Sigma-Aldrich; Merck KGaA, Darmstadt, Germany) and stored at $-20^{\circ} \mathrm{C}$. The primary antibodies for STAT3 (cat. no. ab68153, monoclonal, raised in rabbit, 1:2,000), phosphorylated (p-) STAT3 (Y705; cat. no. ab76315, monoclonal, raised in rabbit, 1:5,000), JNK (cat. no. ab208035, monoclonal, raised in rabbit, 1:1,000), p38 MAPK (cat. no. ab170099, monoclonal, raised in rabbit, 1:1,000), p-JNK (Y185/Y185/Y223; cat. no. ab76572, monoclonal, raised in rabbit, 1:5,000) and p-p38 MAPK (T180/Y182; cat. no. ab195049, monoclonal, raised in rabbit, 1:1,000) were purchased from Abcam (Cambridge, UK). The antibodies against p44/42 MAPK (ERK1/2; cat. no. 4695, monoclonal, raised in rabbit, 1:1,000), p-p44/42 MAPK (p-ERK1/2, T202/Y204; cat. no. 4377, monoclonal, raised in rabbit, 1:1,000), c-Myc (cat. no. 9402, polyclonal, raised in rabbit, 1:1,000), cyclin D1 (cat. no. 2922, polyclonal, raised in rabbit, 1:1,000), survivin (cat. no. 2803, polyclonal, raised in rabbit, 1:1,000), cleaved-PARP (c-PARP, cat. no. 5625, polyclonal, raised in rabbit, 1:1,000), cleaved-caspase 3 (c-caspase 3 , cat. no. 9661, polyclonal, raised in rabbit, 1:1,000) and
BIM (cat. no. 2933, polyclonal, raised in rabbit, 1:1,000) were purchased from Cell Signaling Technology, Inc. (Beverly, MA, USA). GAPDH (cat. no. HRP-60004, 1:1,000) and HRP-conjugated secondary antibodies (cat. no. SA00001-2, 1:5,000) were purchased from ProteinTech Group, Inc. (Wuhan, China).

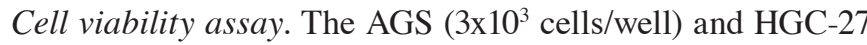
$\left(2 \times 10^{3}\right.$ cells/well $)$ cells were seeded into 96 -well plates, exposed to DMSO vehicle $(1 \mu \mathrm{M})$ or various concentrations of BP-1-102 (2, 4 and $6 \mu \mathrm{M}$ in $1 \mu \mathrm{M}$ DMSO). The maximum final concentration of DMSO was $\leq 0.1 \%$ in the cell culture medium. Following incubation for 24,48 and $72 \mathrm{~h}$ at $37^{\circ} \mathrm{C}$, a Cell Counting Kit-8 (CCK8; Dojindo Molecular Technologies, Inc., Kumamato, Japan) was used to assess cell viability following the manufacturer's protocol, and the absorbance at a wavelength of $450 \mathrm{~nm}$ was measured using a microplate enzyme-linked immunosorbent assay reader (Bio-Rad Laboratories, Inc., Hercules, CA, USA).

Colony formation assay. The AGS $\left(5 \times 10^{2}\right.$ cells/well) and HGC-27 ( $8 \times 10^{2}$ cells/well) cells were seeded in 6-well culture plates, treated with different concentrations of BP-1-102 (2, 4 and $6 \mu \mathrm{M}$ in $1 \mu \mathrm{M}$ DMSO) or DMSO vehicle $(1 \mu \mathrm{M})$. Following culture for $\sim 14$ days, the colonies were fixed with $95 \%$ ethanol, stained with $0.1 \%$ crystal violet for $30 \mathrm{~min}$ and washed with phosphate-buffered saline, following which colony numbers were counted using an inverted microscope (magnification, x200; Zeiss GmbH, Jena, Germany).

Flow cytometry. Apoptosis was evaluated using the Annexin V-fluorescein isothiocyanate (FITC)/propidium iodide (PI) Detection kit (BD Biosciences, San Jose, CA, USA). The AGS cells $\left(2 \times 10^{5}\right.$ cells/well $)$ were seeded into 6-well plates and incubated overnight, following which the cells were treated with the different concentrations of BP-1-102 for $8 \mathrm{~h}$. The cells were then harvested and resuspended in $500 \mu \mathrm{l}$ of $1 \mathrm{X}$ binding buffer solution, incubated with Annexin V-FITC (5 $\mu \mathrm{l})$ and PI $(5 \mu \mathrm{l})$ at $4^{\circ} \mathrm{C}$ for $15 \mathrm{~min}$. Subsequently, the samples were analyzed within $1 \mathrm{~h}$ by flow cytometry (BD Biosciences) and BD CellQuest Pro software (version 2.0, BD Pharmingen; BD Biosciences).

For cell cycle analysis, the BP-1-102-pretreated cells were trypsinized, fixed in $75 \%$ ethanol, incubated at $4^{\circ} \mathrm{C}$ overnight, and then centrifuged at $800 \mathrm{xg}$ for $5 \mathrm{~min}$ at room temperature. The cells were then re-suspended in PI and RNase A solution for $30 \mathrm{~min}$ at room temperature in the dark and evaluated using flow cytometry (BD Biosciences) and BD CellQuest Pro software (BD Pharmingen; BD Biosciences) within $1 \mathrm{~h}$.

Transwell assay for migration and invasion. For the migration and invasion assays, the cells were pre-exposed to BP-1-102 $(6 \mu \mathrm{M}$ in $1 \mu \mathrm{M}$ DMSO) or DMSO vehicle $(1 \mu \mathrm{M})$ for $8 \mathrm{~h}$ and 24-well Transwell ${ }^{\circledR}$ plates with 8 - $\mu$ m pore polycarbonate filters (Costar; Corning Incorporated, Corning, NY, USA) were used. The cells were harvested in serum-free RPMI-1640 medium at a density of $5 \times 10^{4}$ cells in $200 \mu 1$ and seeded into the upper chambers, which had either been coated with Matrigel (BD Biosciences) or left uncoated. Subsequently, $700 \mu \mathrm{l}$ of RPMI-1640 complete medium was added to the 
A

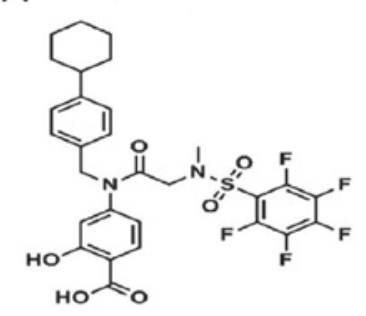

B

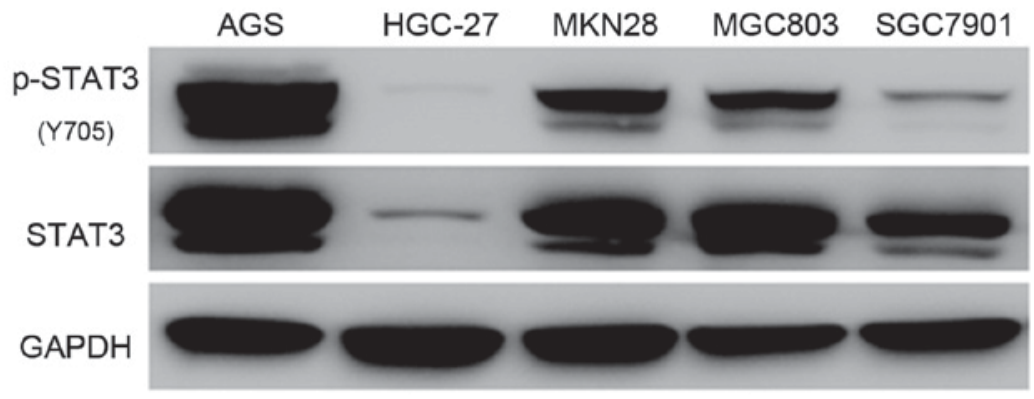

Figure 1. Expression status of STAT3 in GC cell lines. (A) Chemical structure of BP-1-102. (B) Constitutive expression of STAT3 and p-STAT3 (Y 705) in five GC cell lines (AGS, HGC-27, MKNK28, MGC803 and SGC7901). STAT3, signal transducer and activator of transcription 3; p-, phosphorylated.

lower chambers. Following incubation for $18 \mathrm{~h}$, the cells that had penetrated the membrane into the lower chamber were fixed with $95 \%$ ethanol and stained with $0.1 \%$ crystal violet. Images were then captured with a light microscope under x200 magnification.

Western blot analysis. The cells $\left(5 \times 10^{5}\right.$ cells/well) were cultured in six-well plates. Following treatment with BP-1-102 $(6 \mu \mathrm{M})$ for various durations $(0,3,5$ and $8 \mathrm{~h})$ or at various concentrations of BP-1-102 $(0,2,4$ and $6 \mu \mathrm{M})$ for $8 \mathrm{~h}$, the cells were harvested and quantified as previously described (22). Samples containing $20 \mu \mathrm{g}$ of protein were subjected to electrophoresis on 10 or $12 \%$ SDS-PAGE gels and transferred onto a polyvinylidene difluoride membrane (EMD Millipore, Billerica, MA, USA). The membranes were blocked using blocking buffer $(10 \%$ skimmed milk in Tris-buffered saline containing $0.1 \%$ Tween-20) for $1 \mathrm{~h}$, incubated with primary antibodies overnight at $4^{\circ} \mathrm{C}$, and then incubated with anti-rabbit HRP-conjugated secondary antibodies. Finally, the immunoreactive protein bands were visualized using an enhanced chemiluminescence western blotting kit (Bio-Rad Laboratories, Inc.) following the manufacturer's protocol.

Statistical analysis. Experimental data from experiments conducted in triplicate are presented as the mean \pm standard deviation. Statistical analyses between two groups were performed using Student's t-test, and multiple comparisons were made using one-way analysis of variance followed by Dunnett's test (GraphPad Prism 5.01; GraphPad Software, Inc., San Diego, CA, USA). $\mathrm{P}<0.05$ was considered to indicate a statistically significant difference.

\section{Results}

BP-1-102 inhibits the growth of AGS cells. The chemical structure of BP-1-102 is shown in Fig. 1A. To evaluate the antitumor effect of BP-1-102, five GC cell-lines (AGS, HGC-27, MKN28, MGC803 and SGC7901) were used to examine the expression status and the phosphorylation level of STAT3. The AGS cells exhibited a high level of STAT3 Y705 phosphorylation, which is the most studied activated-form of STAT3, whereas the HGC-27 cells exhibited the lowest expression profile (Fig. 1B). Based on these results, the AGS and HGC-27 cell lines were selected for subsequent experiments. The AGS and HGC-27 cells were exposed to various concentrations of BP-1-102 to evaluate its effect on the proliferation of GC cells using a CCK8 assay. Compared with the control group, BP-1-102 treatment dose-dependently suppressed the proliferation of AGS cells (Fig. 2A) but had no such inhibitory effect on HGC-27 cells (Fig. 2B). Furthermore, the results of the colony formation assays showed that the BP-1-102-treated AGS cells formed smaller and fewer colonies compared with those in the control group. BP-1-102 was less effective towards HGC-27 cells than AGS cells (Fig. 2C and D). These results indicated that BP-1-102 exerted a tumor suppressive role in GC cells lines and that this effect was enhanced by high expression levels of p-STAT3 (Y705). Therefore, the AGS cell line was selected for subsequent experiments.

BP-1-102 induces apoptosis in AGS cells and has little effect on cell cycle. The Annexin V-FITC/PI double staining assay was performed to investigate whether the antitumor effect of BP-1-102 was associated with the induction of apoptosis. As shown in Fig. 3A and B, the proportion of apoptotic AGS cells (Annexin V-FITC positive) increased from $7.6 \%$ in the untreated control cells to $8.37,11.04$ and $13.91 \%$ following treatment for $8 \mathrm{~h}$ with 2, 4 and $6 \mu \mathrm{M}$ of BP-1-102, respectively. The expression of apoptotic-related proteins was analyzed by western blotting. As shown in Fig. 3C, BP-1-102 treatment markedly increased the expression of cleaved-poly (ADP-ribose) polymerase (PARP), cleaved-caspase 3, and B-cell lymphoma 2 (Bcl-2)-interacting mediator of cell death (Bim) in a dose-dependent manner. BP-1-102 treatment had no significant influence on the AGS cell cycle distribution (Fig. 3D and E).

BP-1-102 inhibits the mobility of AGS cells. Transwell assays were conducted to assess the effect of BP-1-102 on tumor cell migration and invasion. As shown in Fig. 4, the number of penetrating cells was significantly lower in the BP-1-102-treated group $(6 \mu \mathrm{M})$ compared with that in the control group (untreated). These data indicate the involvement of BP-1-102 in suppressing the mobility of AGS cells.

BP-1-102 inhibits the phosphorylation of STAT3 and its target genes in a time-dependent and dose-dependent manner. The previous findings demonstrated that BP-1-102 inhibited cell growth, migration and invasion ability and induced apoptosis. To gain insight into the potential mechanism of BP-1-102 activity in AGS cells, the activation of STAT3 and 
A

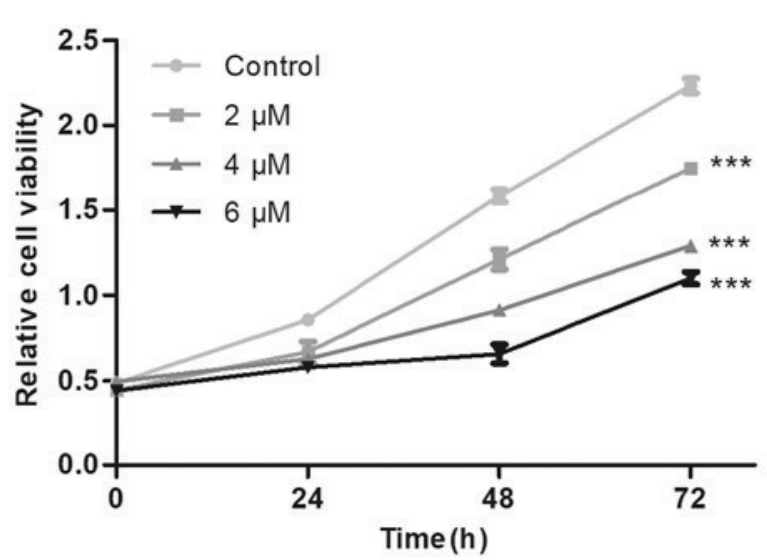

C

\section{Control}
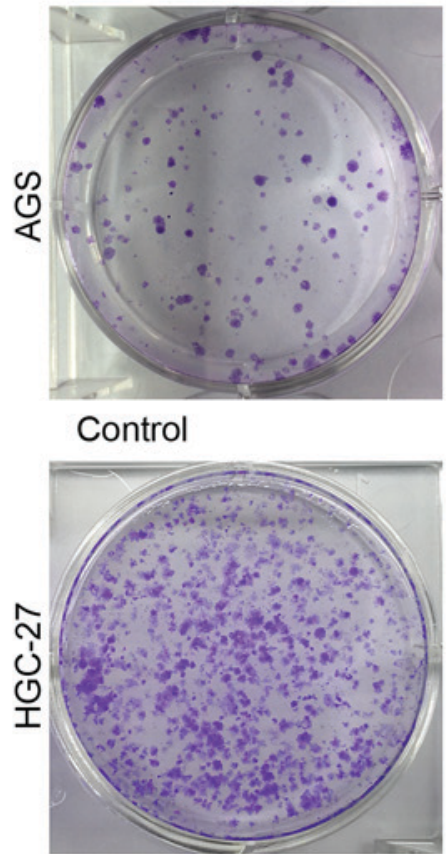

$2 \mu \mathrm{M}$

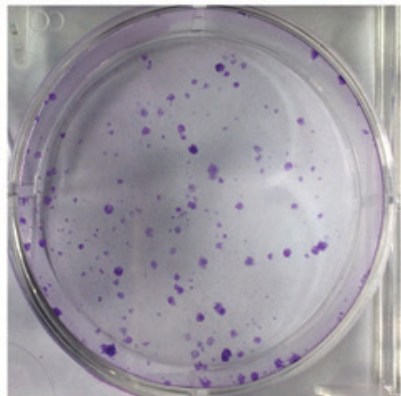

\section{$2 \mu \mathrm{M}$}

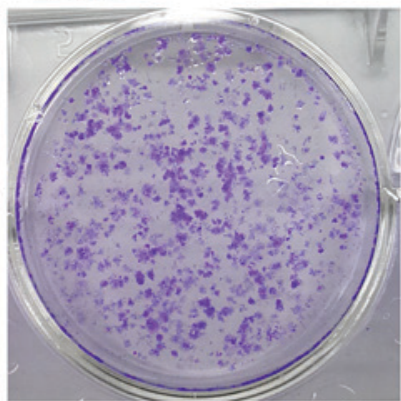

B

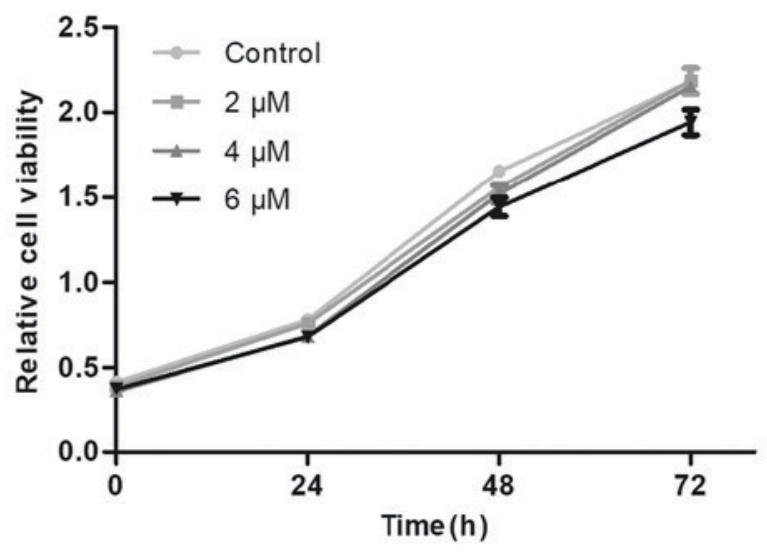

$4 \mu \mathrm{M}$

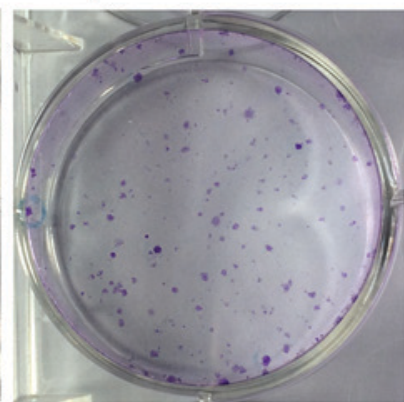

$4 \mu \mathrm{M}$

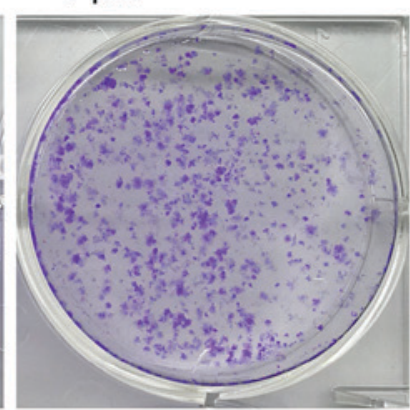

$6 \mu \mathrm{M}$

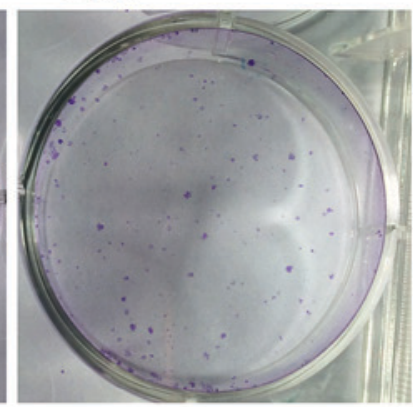

$6 \mu \mathrm{M}$

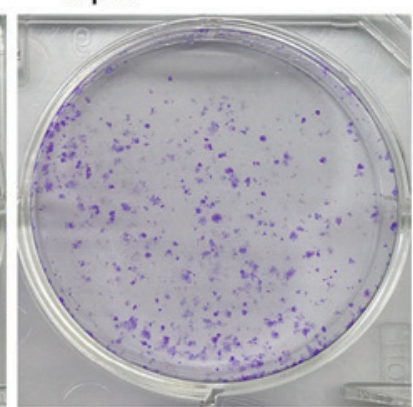

D
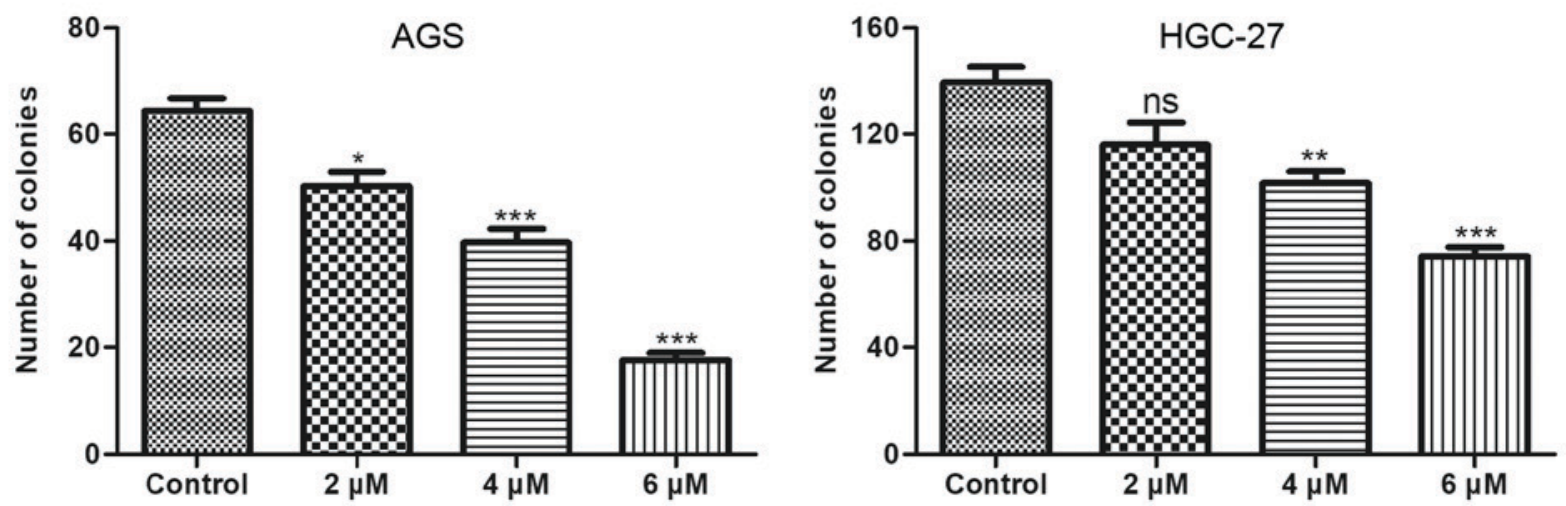

Figure 2. Inhibitory effects of BP-1-102 on the proliferation of AGS cells. Cell viability, determined by Cell Counting Kit-8, of (A) AGS and (B) HGC-27 cells treated with different concentrations of BP-1-102 for $72 \mathrm{~h}$. (C) AGS and HGC-27 cells were treated with BP-1-102 at 2-6 $\mu$ M and cultured for $\sim 14$ days until the colonies were visible. (D) Histograms of the number of cells in each treatment group in AGS (left) and HGC-27 (right) cells. Data were obtained from three independent experiments. ${ }^{*} \mathrm{P}<0.05,{ }^{* *} \mathrm{P}<0.01$ and ${ }^{* * *} \mathrm{P}<0.001$, vs. control. ns, not significant.

its target genes were examined using western blot analysis. As shown in Fig. 5A, the expression of p-STAT3 (Y705) was markedly suppressed by BP-1-102 treatment in a time- and dose-dependent manner, whereas the expression levels of total STAT3 remained unchanged. The STAT3 signaling pathway regulates cell proliferation and survival by modulating the 
A
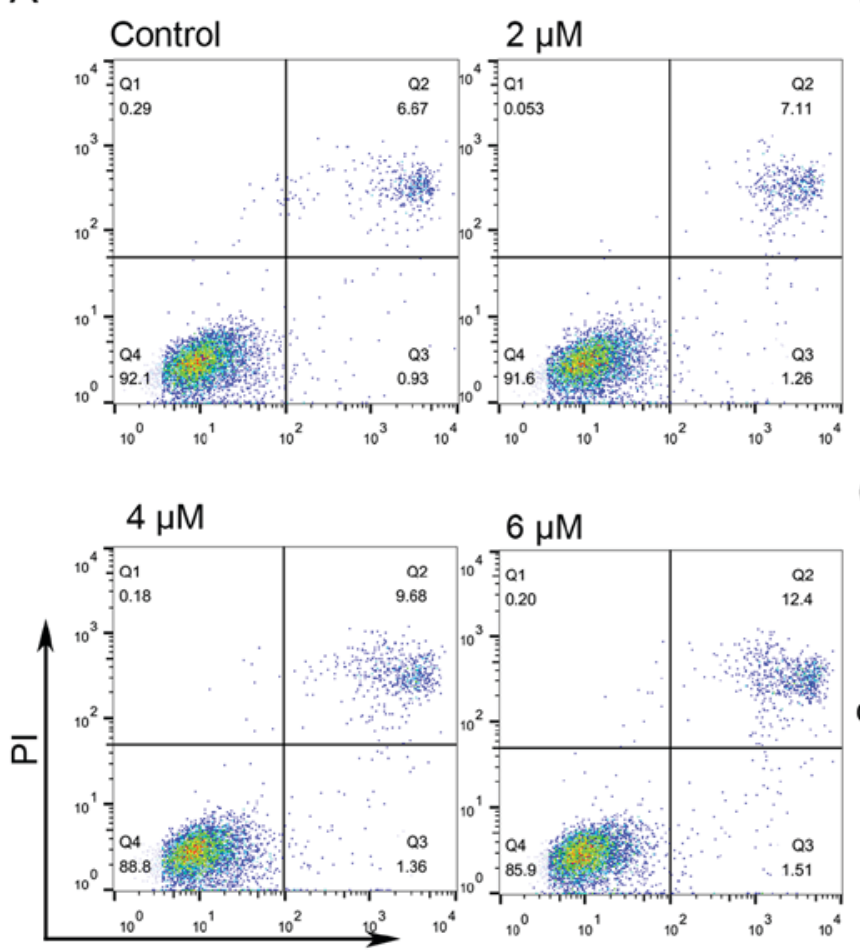

Annexin-V-FITC

$\mathrm{D}$
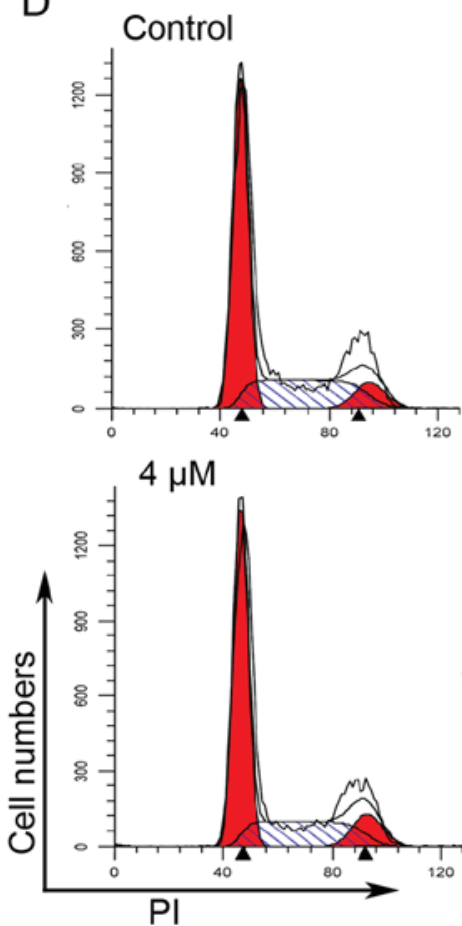

B

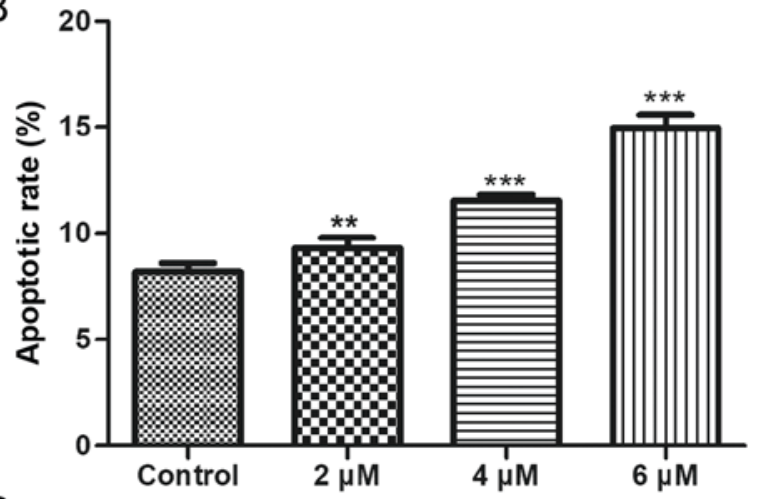

C
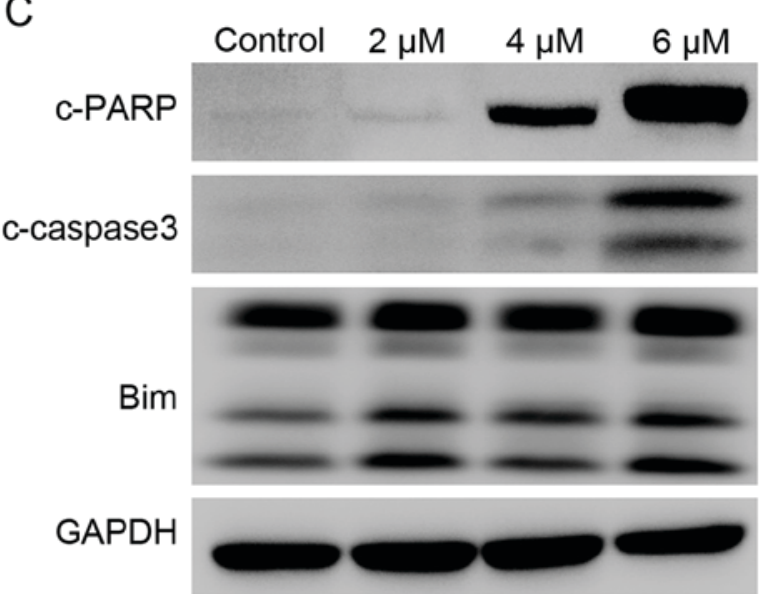

E

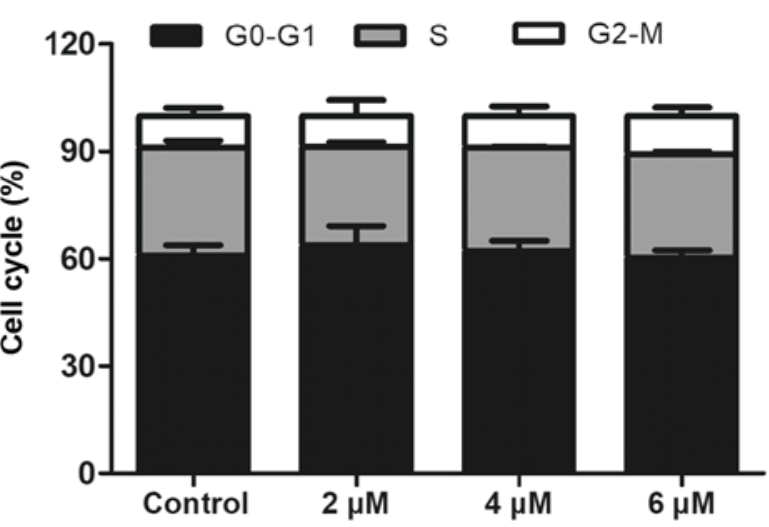

Figure 3. BP-1-102 induces apoptosis in AGS cells and has little effect on cell cycle. (A) Annexin V and PI double staining of AGS cells was performed using flow cytometry to evaluate the apoptotic cells in AGS following exposure to different concentrations of BP-1-102 for 8 h. (B) Histograms of the percentage of apoptotic cells. (C) Western blot analysis of apoptosis-related proteins. GAPDH was used as a protein loading control. (D) PI staining and flow cytometric analysis of AGS cells pretreated with different concentrations of BP-1-102 for $8 \mathrm{~h}$. (E) Cell cycle distribution shown as histograms. The results are representative of three independent experiments. ${ }^{* *} \mathrm{P}<0.01$ and ${ }^{* * * *} \mathrm{P}<0.001$, vs. control. PARP, poly (ADP) ribose polymerase; Bim, B-cell lymphoma-2-interacting mediator of cell death; c-, cleaved; PI, propidium iodide; FITC, fluorescein isothiocyanate.

expression of various target genes. The results showed that BP-1-102 also time- and dose-dependently downregulated the expression of c-Myc, cyclin D1 and survivin (Fig. 5B).
BP-1-102 modulates the expression of MAPKs. As BP-1-102 inhibited cell growth and mobility and induced apoptosis, the possible mechanisms involved were investigated by evaluating 

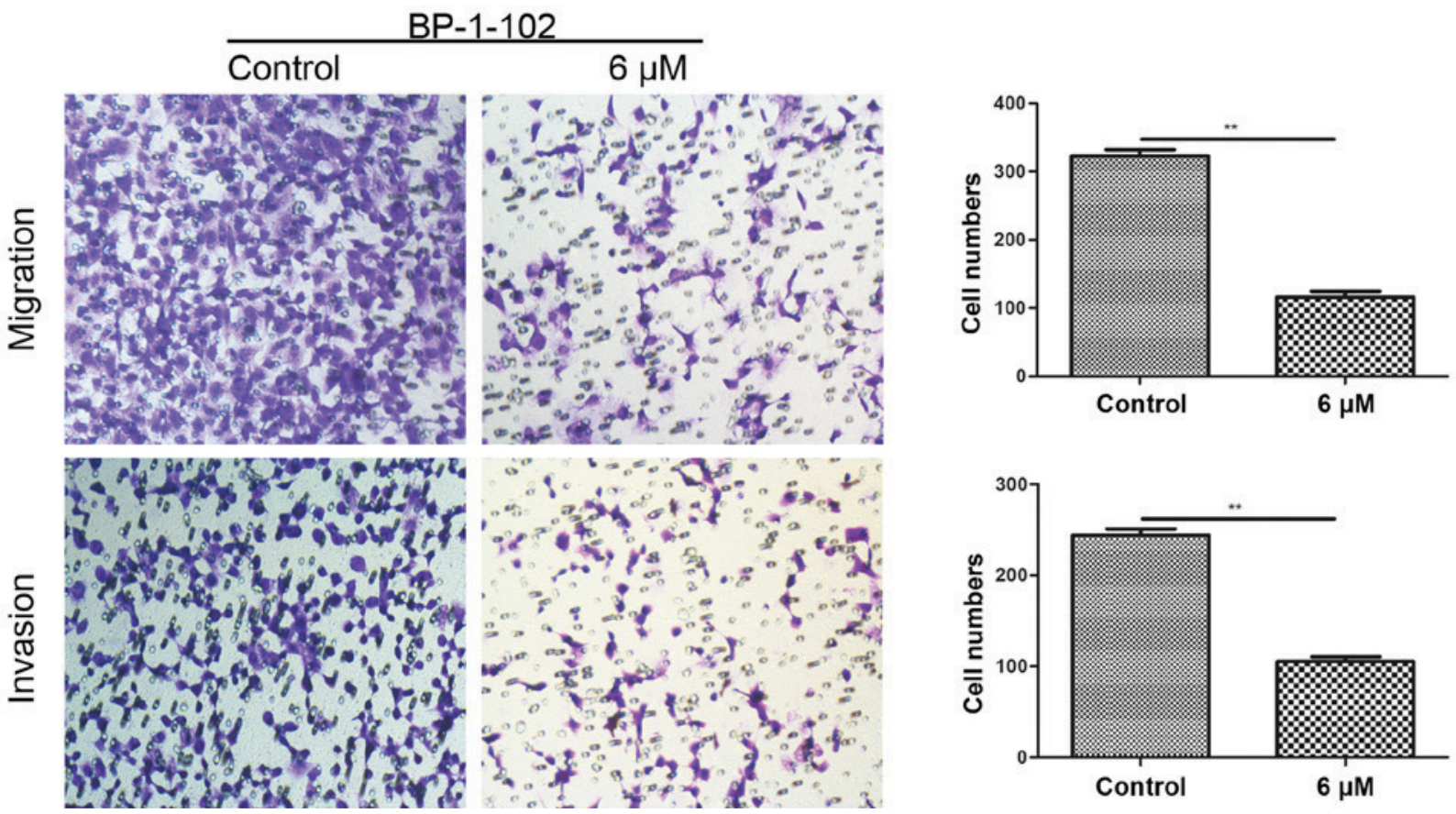

Figure 4. BP-1-102 suppresses the migration and invasion of AGS cells. Transwell assay showed that BP-1-102 markedly attenuated the migration (without Matrigel) and invasion capacity (with Matrigel) of AGS cells. Following pre-exposure to BP-1-102 for $8 \mathrm{~h}$, the cells were seeded in the top compartment of the Transwell chambers and incubated for $18 \mathrm{~h}$ (magnification, x200). The numbers of migrated and invaded cells were quantified from three independent experiments. ${ }^{* *} \mathrm{P}<0.01$, vs. control.

A

\begin{tabular}{llll}
$A$ & \multicolumn{4}{c}{ BP-1-102 $(6 \mu \mathrm{M})$} \\
\hline 0 & 3 & 5 & $8 \mathrm{~h}$
\end{tabular}
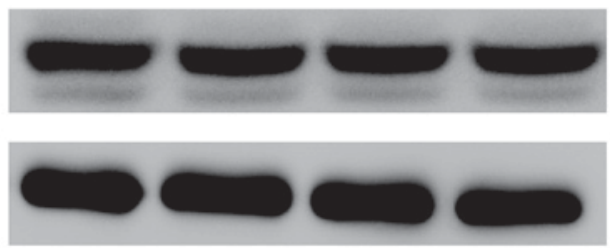

GAPDH

STAT3
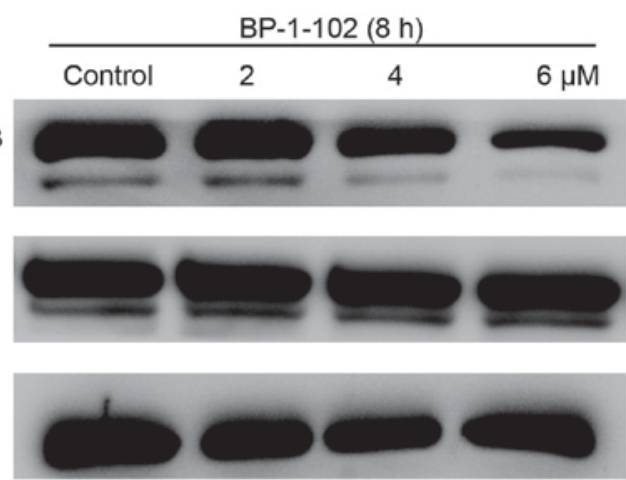

BP-1-102 $(8 \mathrm{~h}$

\begin{tabular}{llcc}
\multicolumn{4}{c}{ BP-1-102 (8h) } \\
\hline Control & 2 & 4 & $6 \mu \mathrm{M}$
\end{tabular}

Cyclin D1

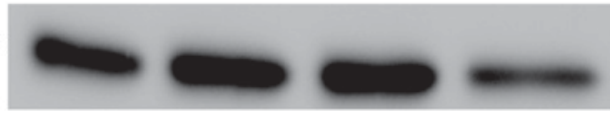

Survivin

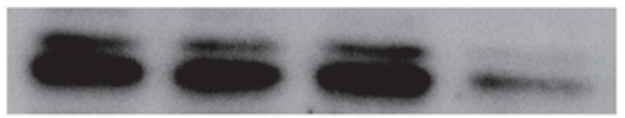

GAPDH

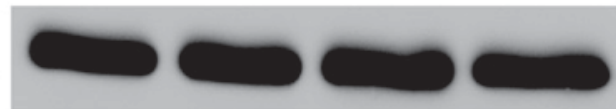

Figure 5. BP-1-102 inhibits the phosphorylation of STAT3 and downregulates the expression of STAT3-regulated genes in AGS cells in a time- and dose-dependent manner. (A) Western blot analysis of p-STAT3 and STAT3 in AGS cells following treatment with $6 \mu \mathrm{M}$ BP-1-102 for various durations (left) or with different concentrations of BP-1-102 for $8 \mathrm{~h}$ (right). (B) BP-1-102 time-dependently (left) and dose-dependently (right) decreased the expression of c-Myc, cyclin D1 and survivin in AGS cells. The results are representative of three independent experiments. STAT3, signal transducer and activator of transcription 3; p-, phosphorylated. 


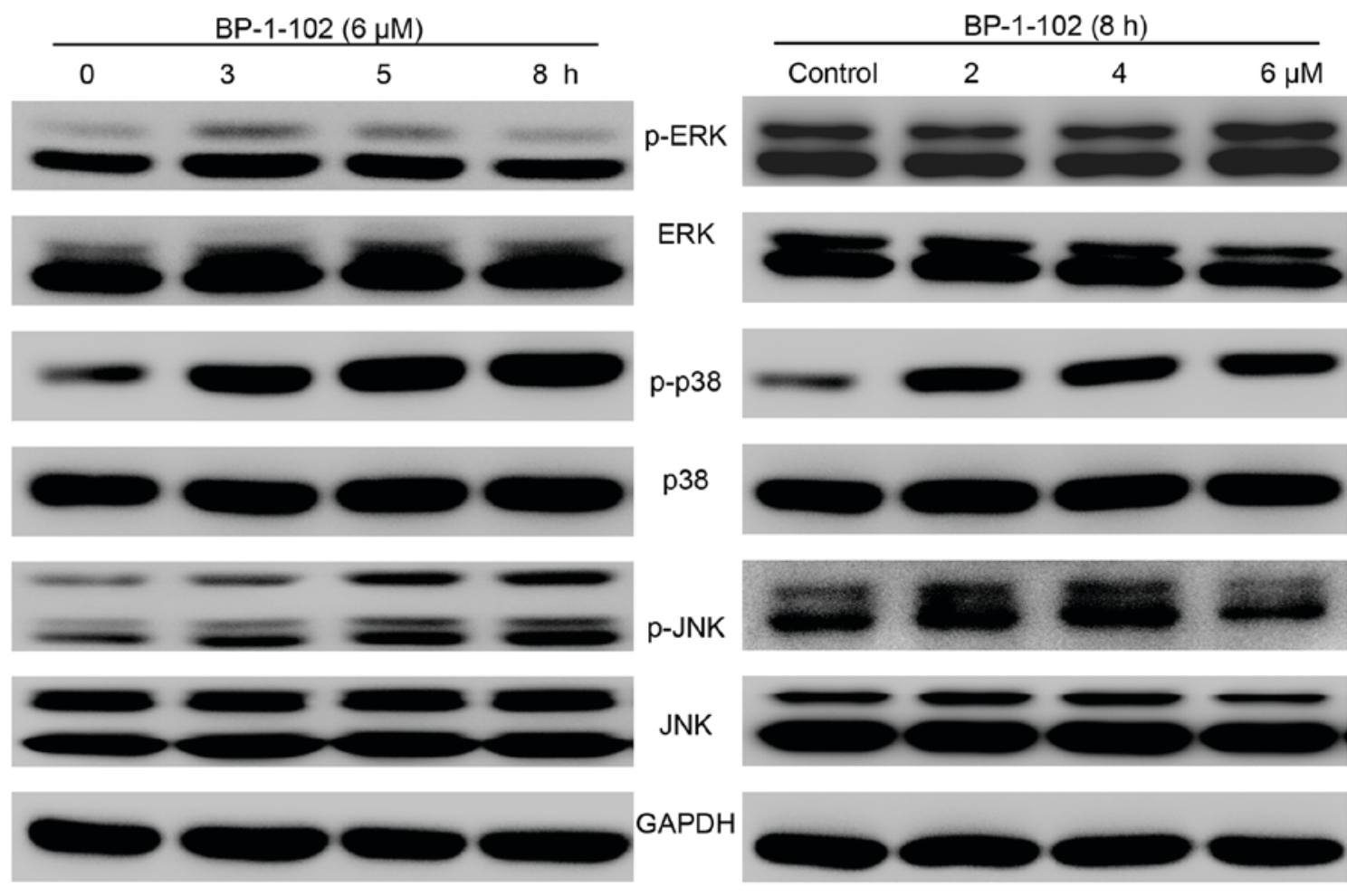

Figure 6. Effects of BP-1-102 on MAPKs. Western blot analysis was used to evaluate the expression of MAPKs (p-ERK, ERK, p-p38, p38, p-JNK and JNK). Cells were treated with the indicated drug concentrations for different durations (right) or with indicated concentrations of BP-1-102 for $8 \mathrm{~h}$ (right). The results are representative of at least three independent experiments. MAPK, mitogen-activated protein kinase; ERK, extracellular signal-regulated kinase; JNK, c-Jun NH2-terminal kinase; p-, phosphorylated.

the effect of BP-1-102 treatment on the elementary activation status of MAPKs. As shown in Fig. 6, following exposure of the AGS cells to BP-1-102, the phosphorylation of p38 and JNK was upregulated, whereas the phosphorylation of ERK was decreased, in a time- and dose-dependent manner relative to the total levels of p38, JNK and ERK, which served as internal controls.

\section{Discussion}

STAT3 is pivotal in signal transduction and transcription, and regulates the transcriptional activity of downstream target genes involved in cell proliferation (c-Myc and cyclin D1), anti-apoptotic genes (Bcl-2, Bcl-extra large and survivin) and genes involved in invasion (matrix metalloproteinase-2 and -9) $(23,24)$. Although the basal expression levels differ in tissues from different organs, STAT3 is frequently overexpressed or constitutively activated in human solid tumors compared with matched normal tissues (25). Previously, constitutively activated STAT3 was reported to occur in various types of cancer, including prostate, renal, breast, ovarian and liver cancer, and is correlated with poor overall survival rates of patients with lung cancer or GC (26-28). In particular, a previous study of $\mathrm{GC}$ found that the overexpression levels of p-STAT3 and total STAT3 were correlated with poor overall survival rate (29). Therefore, although the role of STAT3 in tumorigenesis remains to be fully elucidated, the inhibition of STAT3 continues to be an attractive strategy for the clinical treatment of tumors with activated STAT3.
Several strategies have been established for the inhibition of STAT3, including the inhibition of upstream activators, inhibiting the phosphorylation of STAT3 itself, and suppressing the nuclear translocation of STAT3; notably a large number of inhibitors targeting STAT3 have been identified over the last few decades $(14,30)$. Specifically, several non-peptide STAT3-SH2 domain inhibitors, including S3I-201, STATTIC and STA-21, have been found to be effective in suppressing cell growth in vitro and in vivo (31-33). S3I-201 (NSC 74859) was effective in inhibiting the formation of STAT3 dimers $\left(\mathrm{IC}_{50}=86 \mu \mathrm{M}\right)$ and the expression of STAT3 target genes (c-Myc, cyclin D1 and survivin) in breast cancer (33). In liver cancer, it was found that S3I-201 was not only able to inhibit tumor growth but also significantly enhanced the antitumor effects of cetuximab and doxorubicin (34-36). STATTIC can selectively inhibit STAT3 dimerization $\left(\mathrm{IC}_{50}=5.1 \mu \mathrm{M}\right)$ and nuclear translocation in breast and liver cancer (31). In addition, it has been found that STATTIC enhances chemo- and radio-sensitivity in patients with head and neck squamous cell carcinoma $(37,38)$. STA-21 was found to reduce the survival of breast carcinoma cells with hyperactivated STAT3; however, it exerted little effect on cells with a low expression of activated STAT3 (32). STA-21 has been also reported to be involved in regulating cell differentiation and inflammation (39-41). However, although present inhibitors have an acceptable antitumor effect, they are neither effective at low concentrations nor orally bioavailable. For these reasons, although substantial progress has been made, further progress is necessary, and novel inhibitors with higher efficiency, improved bioavailability and fewer side effects are required. BP-1-102 is a novel orally bioavailable 
STAT3 inhibitor that functions by directly interacting with STAT3 at a relatively low concentration $\left(\mathrm{IC}_{50}=6.4 \mu \mathrm{M}\right)$ and has been shown to have significant antitumor effects in several tumors (19); however, its potential use in GC treatment has not been investigated previously.

In the present study, cell growth, colony formation, apoptosis, cell cycle and cell mobility were evaluated in vitro to determine the effect of BP-1-102 on the biological characteristics of AGS cells. It was found for the first time, to the best of our knowledge, that BP-1-102 exhibited superior suppressive effects on the cell proliferation and colony formation capacities of AGS cells with a higher expression level of p-STAT3 (Y705) than HGC-27 cells with lower expression levels of p-STAT3 (Y705). In addition, the expression status of STAT3 and several STAT3-regulated genes were examined. The results revealed that the constitutive activation of STAT3 was inhibited by BP-1-102 in a time- and dose-dependent manner. Subsequently, the expression levels of c-Myc and cyclin D1, two proteins associated with the inhibition of cell proliferation, decreased when the cells were exposed to BP-1-102. Apoptosis assays demonstrated that the proportion of apoptotic cells increased upon incubation of the cells with BP-1-102, which can be attributed to suppression of the anti-apoptotic protein surviving (42). Additionally, several apoptotic-related proteins were measured via western blotting. When exposed to BP-1-102, the expression of cleaved-PARP, cleaved-caspase 3 and pro-apoptotic Bim were markedly increased in the AGS cells in a dose-dependent manner. However, although a significant decrease in the expression of cyclin D1, an important target in regulating cell cycle (43), was observed, the cell cycle distribution of AGS cells remained unchanged when pretreated with BP-1-102 for $8 \mathrm{~h}$. In addition, based on the results of the Transwell assays, the migration and invasion capacities of AGS cells were markedly suppressed by BP-1-102 treatment. The detailed mechanisms underlying these effects of BP-1-102 on AGS cells remain to be elucidated.

The dysregulation of MAPK is critical in tumor development $(18,44)$. ERK, the most widely studied MAPK, has been shown to be a major regulator in cell growth and the activation of p38 and JNK MAPKs are reported to be positively correlated with apoptosis $(16,45,46)$. In the present study, BP-1-102 was found to activate JNK and p38 MAPK and to inactivate ERK in AGS cells in a time- and dose-dependent manner. The results suggest that BP-1-102 may also act as a MAPK regulator in AGS cells. However, future investigations are warranted to further examine the roles of MAPKs in the effects of BP-1-102 on growth suppression and apoptosis induction in AGS cells. There were several limitations to the present study. For example, the mechanism was based on a single cell line and in vivo experiments are required to further evaluate the antitumor effect and toxicity of BP-1-102. Further experiments, including in vivo experiments are to be performed in the future to validate its antitumor potential in GC.

In conclusion, the present study is the first, to the best of our knowledge, to demonstrate that BP-1-102 is a potent inhibitor of STAT3 signaling and a potential regulator of MAPKs in GC cells. BP-1-102 suppressed the proliferation, migration and invasion capacities of AGS human gastric cancer cells and induced their apoptosis. These findings suggest that BP-1-102 may be a potential therapeutic agent. Future in vivo experiments are warranted to further evaluate this possible approach, and to determine the efficacy and safety of BP-1-102 in treating patients with GC with hyperactivated STAT3.

\section{Acknowledgements}

Not applicable.

\section{Funding}

This study was supported by grants from the National Natural Science Foundation of China (grant nos. 81201089 and 81272676) and the Natural Science Foundation of Zhejiang province, China (grant nos. LY15H160026 and LY15H160012).

\section{Availability of data and materials}

The data used and/or analyzed in the present study are available from the corresponding author on reasonable request.

\section{Authors' contributions}

XXJ, JT, ZQL, XFY and LST conceived and designed the study. XXJ, JT, MJW, STC, ZZX, YHW and HHW performed the data analysis. XXJ and JT wrote the paper. All the authors reviewed and edited the manuscript. All authors read and approved the manuscript.

\section{Ethics approval and consent to participate}

Not applicable.

\section{Patient consent for publication}

Not applicable.

\section{Competing interests}

The authors declare that they have no competing interests.

\section{References}

1. Siegel RL, Miller KD and Jemal A: Cancer Statistics, 2017. CA Cancer J Clin 67: 7-30, 2017.

2. Chen W, Zheng R, Baade PD, Zhang S, Zeng H, Bray F, Jemal A, Yu XQ and He J: Cancer statistics in China, 2015. CA Cancer J Clin 66: 115-132, 2016.

3. Jiang C, Chen X, Alattar M, Wei J and Liu H: MicroRNAs in tumorigenesis, metastasis, diagnosis and prognosis of gastric cancer. Cancer Gene Ther 22: 291-301, 2015.

4. Ajani JA: Chemotherapy for gastric carcinoma: New and old options. Oncology (Williston Park) 12: 44-47, 1998.

5. Tannock IF: Cancer: Resistance through repopulation. Nature 517: 152-153, 2015.

6. Kessler DA, Austin RH and Levine H: Resistance to chemotherapy: Patient variability and cellular heterogeneity. Cancer Res 74: 4663-4670, 2014.

7. Judd LM, Bredin K, Kalantzis A, Jenkins BJ, Ernst M and Giraud AS: STAT3 activation regulates growth, inflammation, and vascularization in a mouse model of gastric tumorigenesis. Gastroenterology 131: 1073-1085, 2006.

8. Bromberg JF, Wrzeszczynska MH, Devgan G, Zhao Y, Pestell RG, Albanese C and Darnell JE Jr: Stat 3 as an oncogene. Cell 98: 295-303, 1999. 
9. Li Y, Rogoff HA, Keates S, Gao Y, Murikipudi S, Mikule K, Leggett D, Li W, Pardee AB and Li CJ: Suppression of cancer relapse and metastasis by inhibiting cancer stemness. Proc Natl Acad Sci USA 112: 1839-1844, 2015.

10. Hajimoradi M, Mohammad Hassan Z, Ebrahimi M, Soleimani M, Bakhshi M, Firouzi J and Samani FS: STAT3 is overactivated in gastric cancer stem-like cells. Cell J 17: 617-628, 2016.

11. Deng JY, Sun D, Liu XY, Pan Y and Liang H: STAT-3 correlates with lymph node metastasis and cell survival in gastric cancer. World J Gastroenterol 16: 5380-5387, 2010.

12. Gong W, Wang L, Yao JC, Ajani JA, Wei D, Aldape KD, Xie K, Sawaya R and Huang S: Expression of activated signal transducer and activator of transcription 3 predicts expression of vascular endothelial growth factor in and angiogenic phenotype of human gastric cancer. Clin Cancer Res 11: 1386-1393, 2005.

13. Cafferkey $\mathrm{C}$ and Chau I: Novel STAT 3 inhibitors for treating gastric cancer. Expert Opin Investig Drugs 25: 1023-1031, 2016.

14. Furtek SL, Backos DS, Matheson CJ and Reigan P: Strategies and approaches of targeting STAT3 for cancer treatment. ACS Chem Biol 11: 308-318, 2016 .

15. Lai PS, Rosa DA, Magdy Ali A, Gómez-Biagi RF, Ball DP, Shouksmith AE and Gunning PT: A STAT inhibitor patent review: Progress since 2011. Expert Opin Ther Pat 25: 1397-1421, 2015.

16. Boutros T, Chevet E and Metrakos P: Mitogen-activated protein (MAP) kinase/MAP kinase phosphatase regulation: Roles in cell growth, death, and cancer. Pharmacol Rev 60: 261-310, 2008.

17. Meng $X$ and Zhang S: MAPK cascades in plant disease resistance signaling. Annu Rev Phytopathol 51: 245-266, 2013.

18. Kim EK and Choi EJ: Compromised MAPK signaling in human diseases: An update. Arch Toxicol 89: 867-882, 2015.

19. Page BD, Fletcher S, Yue P, Li Z, Zhang X, Sharmeen S, Datti A, Wrana JL, Trudel S, Schimmer AD, et al: Identification of a non-phosphorylated, cell permeable, small molecule ligand for the Stat3 SH2 domain. Bioorg Med Chem Lett 21: 5605-5609, 2011.

20. Zhang X, Yue P, Page BD, Li T, Zhao W, Namanja AT, Paladino D, Zhao J, Chen Y, Gunning PT and Turkson J: Orally bioavailable small-molecule inhibitor of transcription factor Stat 3 regresses human breast and lung cancer xenografts. Proc Natl Acad Sci USA 109: 9623-9628, 2012

21. Resetca D, Haftchenary S, Gunning PT and Wilson DJ: Changes in signal transducer and activator of transcription 3 (STAT3) dynamics induced by complexation with pharmacological inhibitors of Src homology 2 (SH2) domain dimerization. J Biol Chem 289: 32538-32547, 2014

22. Zheng L, Xu C, Guan Z, Su X, Xu Z, Cao J and Teng L: Galectin-1 mediates TGF- $\beta$-induced transformation from normal fibroblasts into carcinoma-associated fibroblasts and promotes tumor progression in gastric cancer. Am J Transl Res 8: 1641-1658, 2016.

23. Luwor RB, Stylli SS and Kaye AH: The role of Stat 3 in glioblastoma multiforme. J Clin Neurosci 20: 907-911, 2013.

24. Zhang X, Yue P, Fletcher S, Zhao W, Gunning PT and Turkson J: A novel small-molecule disrupts Stat3 SH2 domain-phosphotyrosine interactions and Stat3-dependent tumor processes. Biochem Pharmacol 79: 1398-1409, 2010.

25. Zhuang S: Regulation of STAT signaling by acetylation. Cell Signal 25: 1924-1931, 2013.

26. Tong M, Wang J, Jiang N, Pan H and Li D: Correlation between p-STAT3 overexpression and prognosis in lung cancer: A systematic review and meta-analysis. PLoS One 12: e0182282, 2017.

27. Yu S, Li G, Wang Z, Wang Z, Chen C, Cai S and He Y: The prognostic value of pSTAT3 in gastric cancer: A meta-analysis. J Cancer Res Clin Oncol 142: 649-657, 2016.

28. Aggarwal BB, Sethi G, Ahn KS, Sandur SK, Pandey MK, Kunnumakkara AB, Sung B and Ichikawa H: Targeting signal-transducer-and-activator-of-transcription-3 for prevention and therapy of cancer: Modern target but ancient solution. Ann N Y Acad Sci 1091: 151-169, 2006.

29. Pan YM, Wang CG, Zhu M, Xing R, Cui JT, Li WM, Yu DD, Wang SB, Zhu W, Ye YJ, et al: STAT3 signaling drives EZH2 transcriptional activation and mediates poor prognosis in gastric cancer. Mol Cancer 15: 79, 2016.

30. Gelain A, Mori M, Meneghetti F and Villa S: Signal transducer and activator of transcription protein 3 (STAT3): An update on its direct inhibitors as promising anticancer agents. Curr Med Chem: Jul 19, 2018 (Epub ahead of print)
31. Schust J, Sperl B, Hollis A, Mayer TU and Berg T: Stattic: A small-molecule inhibitor of STAT3 activation and dimerization. Chem Biol 13: 1235-1242, 2006.

32. Song H, Wang R, Wang S and Lin J: A low-molecular-weight compound discovered through virtual database screening inhibits Stat 3 function in breast cancer cells. Proc Natl Acad Sci USA 102: 4700-4705, 2005.

33. Siddiquee K, Zhang S, Guida WC, Blaskovich MA, Greedy B, Lawrence HR, Yip ML, Jove R, McLaughlin MM, Lawrence NJ, et al: Selective chemical probe inhibitor of Stat3, identified through structure-based virtual screening, induces antitumor activity. Proc Natl Acad Sci USA 104: 7391-7396, 2007.

34. Hu QD, Chen W, Yan TL, Ma T, Chen CL, Liang C, Zhang Q, Xia XF, Liu H, Zhi X, et al: NSC 74859 enhances doxorubicin cytotoxicity via inhibition of epithelial-mesenchymal transition in hepatocellular carcinoma cells. Cancer Lett 325: 207-213, 2012.

35. Chen W, Shen X, Xia X, Xu G, Ma T, Bai X and Liang T: NSC 74859-mediated inhibition of STAT3 enhances the anti-proliferative activity of cetuximab in hepatocellular carcinoma. Liver Int 32: 70-77, 2012.

36. Lin L, Amin R, Gallicano GI, Glasgow E, Jogunoori W, Jessup JM, Zasloff M, Marshall JL, Shetty K, Johnson L, et al: The STAT3 inhibitor NSC 74859 is effective in hepatocellular cancers with disrupted TGF-beta signaling. Oncogene 28: 961-972, 2009.

37. Zhang Q, Zhang C, He J, Guo Q, Hu D, Yang X, Wang J, Kang Y, She R, Wang Z, et al: STAT3 inhibitor stattic enhances radiosensitivity in esophageal squamous cell carcinoma. Tumour Biol 36: 2135-2142, 2015.

38. Pan Y, Zhou F, Zhang R and Claret FX: Stat3 inhibitor Stattic exhibits potent antitumor activity and induces chemo- and radio-sensitivity in nasopharyngeal carcinoma. PLoS One 8: e54565, 2013.

39. Ahmad SF, Ansari MA, Nadeem A, Zoheir KMA, Bakheet SA, Alsaad AMS, Al-Shabanah OA and Attia SM: STA-21, a STAT-3 inhibitor, attenuates the development and progression of inflammation in collagen antibody-induced arthritis. Immunobiology 222: 206-217, 2017.

40. Park JS, Kwok SK, Lim MA, Kim EK, Ryu JG, Kim SM, Oh HJ, Ju JH, Park SH, Kim HY and Cho ML: STA-21, a promising STAT-3 inhibitor that reciprocally regulates Th17 and Treg cells, inhibits osteoclastogenesis in mice and humans and alleviates autoimmune inflammation in an experimental model of rheumatoid arthritis. Arthritis Rheumatol 66: 918-929, 2014.

41. Takaishi M, Yokogawa M, Miyoshi K, Nakajima K, DiGiovanni J and Sano S: STA-21, a stat3 inhibitor, induces differentiation of normal human epidermal keratinocytes and human keratinocyte derived cell lines. J Invest Dermatol 130 (Suppl): S97-S97, 2010.

42. Ambrosini G, Adida C and Altieri DC: A novel anti-apoptosis gene, survivin, expressed in cancer and lymphoma. Nat Med 3: 917-921, 1997.

43. Baldin V, Lukas J, Marcote MJ, Pagano M and Draetta G: Cyclin $\mathrm{D} 1$ is a nuclear protein required for cell cycle progression in G1. Genes Dev 7: 812-821, 1993.

44. Burotto M, Chiou VL, Lee JM and Kohn EC: The MAPK pathway across different malignancies: A new perspective. Cancer 120: 3446-3456, 2014.

45. Chen F: JNK-induced apoptosis, compensatory growth, and cancer stem cells. Cancer Res 72: 379-386, 2012

46. Scuteri A, Galimberti A, Maggioni D, Ravasi M, Pasini S, Nicolini G, Bossi M, Miloso M, Cavaletti G and Tredici G: Role of MAPKs in platinum-induced neuronal apoptosis. Neurotoxicology 30: 312-319, 2009.

This work is licensed under a Creative Commons Attribution-NonCommercial-NoDerivatives 4.0 International (CC BY-NC-ND 4.0) License. 